\title{
Effects of charge, size, and shape-asymmetry on the phase behavior of model electrolytes
}

\author{
Qiliang Yan and Juan J. de Pabloa) \\ Department of Chemical Engineering, University of Wisconsin-Madison, Madison, Wisconsin 53706
}

(Received 5 September 2001; accepted 20 November 2001)

\begin{abstract}
Monte Carlo simulations have been conducted to investigate the phase behavior of 2:1 hard-core electrolyte models. Two distinct cases have been considered: In the first, both bivalent cations and monovalent anions are spherical. In the second, bivalent cations are modeled as rigid dimers composed of two tangent hard spheres, each carrying a positive charge at the center. Critical temperatures and densities have been calculated as a function of the size asymmetry between positive and negative ions. The simulated critical temperature and critical density are strongly influenced by size asymmetry and by the shape of the ions. Changes in the critical constants are traced back to ground-state energy configurations of small ionic clusters. The trends observed in simulations for the critical temperature and density as a function of size asymmetry are shown to contradict the predictions of available theoretical formalisms. (C) 2002 American Institute of Physics. [DOI: 10.1063/1.1435567]
\end{abstract}

\section{INTRODUCTION}

A fundamental understanding of the thermodynamic properties and structural behavior of electrolyte solutions is important in a wide range of disciplines. Simple electrolytes interact through Coulombic forces, and as such they represent one of the simplest systems in which to study the effects of long-range interactions on thermodynamics and structure. One of the most widely used models of electrolytes in solution is the so-called "restricted primitive model" (RPM), in which monovalent, equal-sized hard spheres interact via a Coulombic potential-energy function. The RPM has been studied extensively, both theoretically and by simulations. At low temperatures, this model exhibits a coexistence region which, for many years, eluded theoretical and numerical attempts to describe it. Over the last decade, armed with better techniques and more powerful computers, molecular simulations have gradually provided a coherent picture of the nature and precise location of the critical point of RPM electrolytes. $^{1-3}$

Recent work has gradually shifted towards study of the phase behavior of more comprehensive, less restrictive models of electrolytes; models in which the size of the ions or their valence are allowed to be asymmetric. The recent study by Orkoulas et al. ${ }^{4}$ and our own simulations ${ }^{5,6}$ have provided some of the first glimpses of how the coexistence curve of monovalent electrolytes changes when the cations and anions exhibit a size asymmetry. That work has yielded interesting insights, particularly regarding the tendency of electrolytes to form large polymeric structures at low temperatures, a tendency that is clearly exacerbated with increasing size asymmetry between cations and anions. The formation of such large clusters is not envisaged by most available theoretical formalisms; comparison of simulated critical constants and theoretical predictions for size-asymmetric elec-

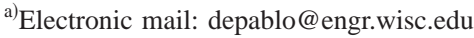

trolytes has revealed that several established theories, such as the mean spherical approximation (MSA), are unable to predict the trends observed in simulations, particularly with regards to the decrease of the critical temperature $T_{c}$ and critical density $\rho_{c}$ with increasing size asymmetry.

Aside from an isolated study by Camp and Patey, ${ }^{7}$ simulations have avoided the more general case of charge asymmetric and size asymmetric electrolytes. In this work, we present the results of Monte Carlo simulations of the phase behavior of 2:1 electrolytes, where we examine the effects of size and shape asymmetry. It is found that this behavior is markedly different from that of monovalent, 1:1 electrolytes, and that shape asymmetry has interesting, hitherto unexpected effects on the structure and thermodynamics of electrolytes.

\section{MODELS AND METHODS}

The electrolyte model examined in this work consists of charged hard spheres interacting via a Coulombic potentialenergy function. Ions are immersed in a dielectric continuum, and they interact according to

$$
U_{i j}=\left\{\begin{array}{l}
+\infty \quad r_{i j} \leqslant \sigma_{i j} \\
\varepsilon z_{i} z_{j} \sigma_{ \pm} / r_{i j} \quad r_{i j}>\sigma_{i j}
\end{array}\right.
$$

where $\sigma_{+}$and $\sigma_{-}$denote the diameter of cations and anions, respectively, where $r_{i j}$ is the distance between site $i$ and site $j$, and where $\sigma_{i j}$ is defined as $\sigma_{i j}=\left(\sigma_{i}+\sigma_{j}\right) / 2 ; \varepsilon$ is the unit energy parameter, defined as $\varepsilon=e^{2} /\left(4 \pi D D_{0} \sigma_{ \pm}\right)$, where $e$ is the charge of the electron, $D_{0}$ is the dielectric permeability of vacuum, and $\sigma_{ \pm}=\left(\sigma_{+}+\sigma_{-}\right) / 2$.

Two variants of this model are considered in this work; these are depicted in Fig. 1. The first variant, referred to as the "spherical model," consists of isolated spherical cations and anions. A total of $N_{+}$cations (of diameter $\sigma_{+}$) carry a positive charge of $+2 ; N_{-}$anions (diameter $\sigma_{-}$) carry a negative charge of -1 . Electro-neutrality is preserved by set- 


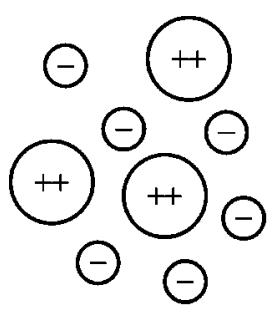

(a)

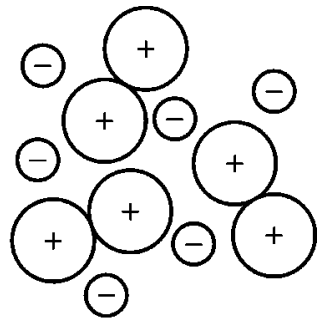

(b)
FIG. 1. Skeletal illustration of two model electrolytes considered in this work. (a) The spherical model; (b) the dumbbell model.

ting $N_{-}=2 N_{+}$. The second variant, referred to as the "dumbbell" model, consists of charged dimers made out of two tangent hard spheres, each having diameter $\sigma_{+}$, and each carrying a positive charge of +1 ; The charge of a dumbbell molecule is +2 . Anions are treated as individual spheres of diameter $\sigma_{-}$and negative charge -1 .

The size asymmetry between cations and anions is quantified by means of parameter $x=\left(\lambda^{\prime}-1\right) /(\lambda+1)$, where $\lambda$ $=\sigma_{+} / \sigma_{-} \cdot{ }^{4-6}$ Throughout this paper, results are reported in reduced units. The reduced temperature is given by $T^{*}$ $=k_{B} T / 2 \varepsilon$, where $k_{B}$ is Bolzmann's constant; the reduced density is given by $\rho^{*}=\left(N_{+}+N_{-}\right) / L^{* 3}$, where $L^{*}$ is a dimensionless box size, defined as $L^{*}=L / \sigma_{ \pm}$.

Monte Carlo simulations were conducted in the framework of a multidimensional parallel tempering formalism in the grand canonical ensemble. In the past, this approach has proven to be effective for simulations of monovalent $(1: 1)$ electrolytes. $3,5,6$ The method has been discussed in detail in the literature; here we only provide a few details to allow others to reproduce our calculations. Depending on the system of interest, a range of 3-5 replicas of the system were simulated; the larger the size-asymmetry, the more replicas were necessary to facilitate sampling of phase space. This is largely due to the lower temperatures involved in the highsize-asymmetry systems.

Depending on size asymmetry, the size of the simulated systems was in the range $L^{*}=12-19$ (the details are reported in Table I); the number of particles (corresponding to the critical density) employed in our calculations was in the range $N_{ \pm}=N_{-}+N_{+}=150-350$. Long-range interactions were calculated using the Ewald-sum method with conducting boundary conditions. Following previous work, ${ }^{5,6}$ the Ewald-sum convergence parameter $\kappa$ was set at $\kappa=5 / L$; for this value of $\kappa, \sim 300 k$-space wave vectors were necessary for the Fourier-space calculation. The real-space cutoff was set at half the simulation box length. Ions were inserted and/or removed from the system in pairs (one cation and two anions), thereby preserving electroneutrality throughout the entire simulation process. A distance-bias sampling scheme was implemented to facilitate such insertions and removals. ${ }^{2}$

The joint distribution of number of ions and total potential energy $p(N, U)$ was estimated in the form of histograms at different temperatures and chemical potentials. Histogram reweighting 8,9 and mixed-field finite-size scaling techniques ${ }^{10,11}$ were subsequently used to combine these histograms and to locate phase boundaries and the apparent
TABLE I. System sizes and critical parameters of 2:1 spherical and dumbbell electrolytes.

\begin{tabular}{|c|c|c|c|}
\hline$x$ & $L^{*}$ & $T_{c}^{*}$ & $\rho_{c}^{*}$ \\
\hline \multicolumn{4}{|c|}{ Spherical Systems } \\
\hline-0.600 & 16.00 & $0.0291(5)$ & $0.037(6)$ \\
\hline-0.333 & 13.33 & $0.0386(4)$ & $0.079(4)$ \\
\hline 0 & 15.00 & $0.0465(3)$ & $0.105(3)$ \\
\hline 0.333 & 13.33 & $0.0469(3)$ & $0.110(4)$ \\
\hline 0.600 & 14.40 & $0.0447(5)$ & $0.103(6)$ \\
\hline 0.818 & 14.55 & $0.0412(6)$ & $0.086(6)$ \\
\hline \multicolumn{4}{|c|}{ Dumbbell Systems } \\
\hline-0.600 & 16.00 & $0.0602(7)$ & $0.072(9)$ \\
\hline-0.471 & 14.71 & $0.0662(5)$ & $0.077(6)$ \\
\hline-0.333 & 13.33 & $0.0717(4)$ & $0.087(4)$ \\
\hline-0.143 & 13.71 & $0.0711(4)$ & $0.097(4)$ \\
\hline 0 & 12.00 & $0.0670(2)$ & $0.103(3)$ \\
\hline 0.143 & 13.71 & $0.0676(3)$ & $0.097(4)$ \\
\hline 0.333 & 16.00 & $0.0675(2)$ & $0.082(3)$ \\
\hline 0.600 & 19.20 & $0.0588(4)$ & $0.074(5)$ \\
\hline 0.778 & 17.78 & $0.0487(6)$ & $0.061(8)$ \\
\hline
\end{tabular}

critical parameters for the finite-size systems. The Ising universality class was assumed in the analysis. The main goal of this work is that of establishing the general, primary trends observed in the critical constants of electrolytes as the charge and size of the ions are changed. As such, no attempt was made to determine system-size effects on the critical parameters.

\section{RESULTS}

Our results for the critical temperature and critical density of 2:1 electrolytes are shown in Fig. 2 and 3, respectively. The numerical data are also reported in Table I. For reference, these figures include results from earlier simulations of 1:1 electrolytes. ${ }^{4-6}$ Our results are consistent with a prediction by Camp and Patey, ${ }^{7}$ who placed the critical temperature of symmetric $(x=0) \quad 2: 1$ electrolytes at $\sim T_{c}^{*}$ $=0.05 \pm 0.005$. Our calculated critical temperatures as a function of $x$ are in quantitative agreement with recent results

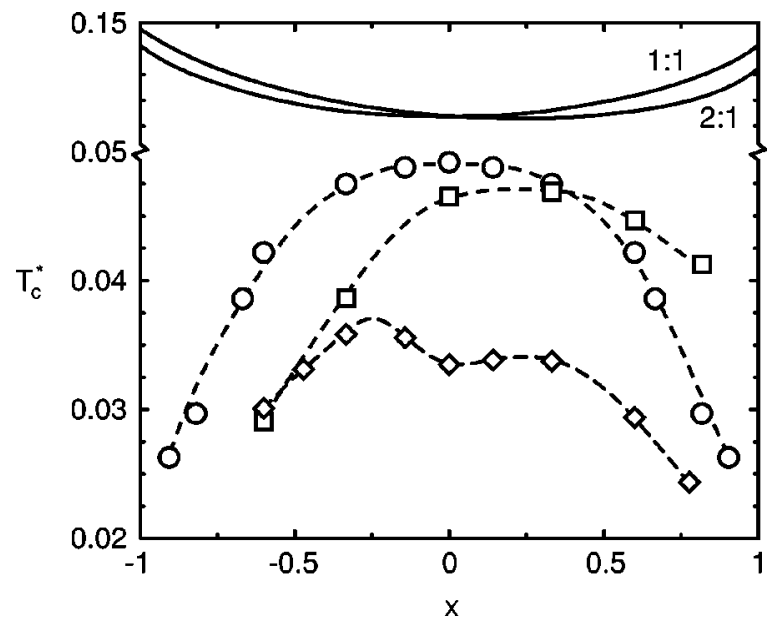

FIG. 2. Critical temperature $T_{c}^{*}$ as a function of size asymmetry. Open symbols correspond to simulation results (squares are 2:1 spherical electrolytes; diamonds are 2:1 dumbbell electrolytes; circles are 1:1 spherical electrolytes $\left.^{3,5}\right)$. The solid lines are MSA results. ${ }^{13}$ 


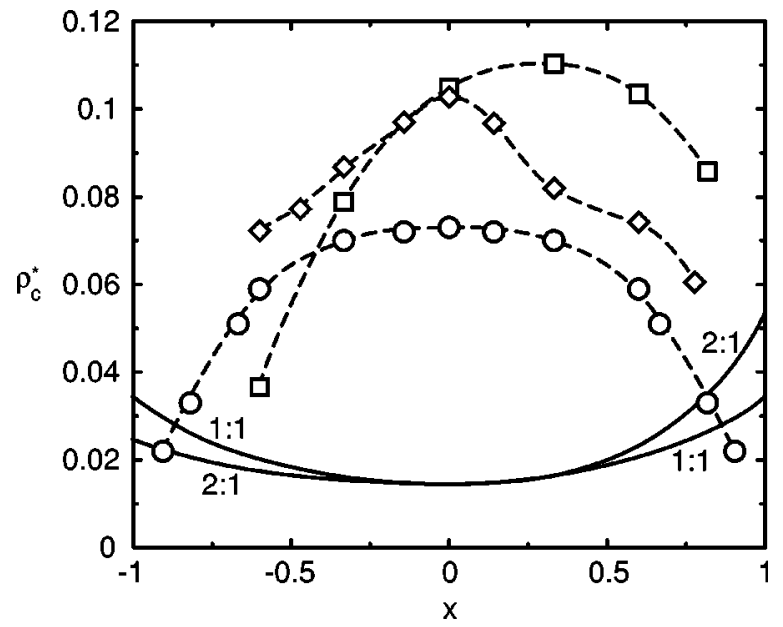

FIG. 3. Critical density $\rho_{c}^{*}$ as a function of size asymmetry. The meaning of the symbols is the same as in Fig. 2.

by Panagiotopoulos and Fisher, who conducted Monte Carlo simulations of the same model but on a finely discretized lattice. ${ }^{12}$ Our calculated critical densities deviate slightly from those of these authors. The differences are small (generally less than 10\%), and they do not appear to be of relevance to the overall conclusions of their study and ours. They are, however, statistically significant. We attribute them to the discretization of phase space involved in the finelattice technique, where the cross-interaction diameter $\sigma_{ \pm}$is mapped onto 10 lattice sites. As mentioned earlier, simulations of asymmetric electrolytes are particularly challenging; we are encouraged by the fact that independent estimates of the critical constants by different groups are comparable and consistent with each other. ${ }^{7,12}$

The critical temperature and density of monovalent, $1: 1$ electrolytes exhibits a maximum at $x=0 .{ }^{4-6}$ Divalent, 2:1 spherical systems also exhibit maxima in the critical temperature and density, but these are shifted towards nonzero values of $x$. The simulations reveal a pronounced sensitivity to $x$. To the best of our knowledge, only the MSA theory has been used to examine the effect of size asymmetry on critical constants; ${ }^{13}$ the predicted changes in $T_{c}^{*}$ and $\rho_{c}^{*}$ with charge asymmetry appear to be relatively minor. Furthermore, the MSA theory indicates that both critical temperature and critical density exhibit a minimum when plotted as a function of size asymmetry.

Other available theoretical predictions for $x=0$ using integral equations ${ }^{14}$ anticipate slight changes of $T_{c}^{*}$ above or below that of the 1:1 system (of at most 2\%). A different, field theoretic approach (also for $x=0)^{15}$ predicts an increase of $T_{c}^{*}$ by a factor of about 2.8 (above the 1:1 system). In contrast, the results of our simulations for $x=0$ indicate that the critical temperature of electrolytes decreases by about 5\% in going from 1:1 to 2:1 systems, and the critical density increases by about $40 \%$.

The maximum in the critical parameters of 1:1 systems occurs precisely at $x=0$; the maximum in the critical parameters of 2:1 systems is shifted towards a positive value of $x$. The effect of size asymmetry is that of stabilizing systems consisting of small divalent cations surrounded by large

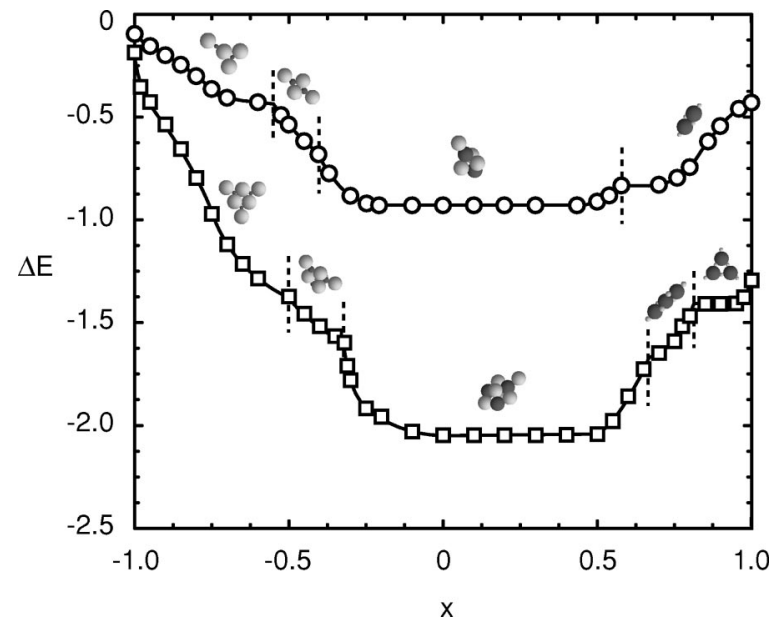

FIG. 4. Ground-state energy of ionic clusters. Circles and squares correspond to two-triplet and three-triplet spherical clusters, respectively. Regions of size asymmetry having distinct ground-state configurations are separated by dashed lines; these configurations are depicted above the curves.

monovalent counterions. Electrolyte systems consisting of large divalent cations surrounded by smaller monovalent counterions phase separate much more readily (i.e., at higher temperatures). Following a line of arguments employed for $1: 1$ systems, $^{6}$ it is instructive to interpret these changes of critical constants with $x$ in terms of energetically favorable arrangements of ions into neutral clusters.

Figure 4 shows the energy change associated with bringing together two or three colinear neutral ion triplets to form a tight minimum-energy cluster. The triplets are originally isolated, and they are brought into contact from a state of infinite separation. The ground-state energies and configurations are determined from zero-temperature Monte Carlo simulations starting from a wide range of initial configurations. Figure 4 also shows these configurations; the geometry and energy of individual clusters depend strongly on size asymmetry. The resulting ground-state energy curves are not symmetric about $x=0$. For two ion triplets, the largest energy drop is attained in the range $x=[-0.21 ; 0.44]$; for three ion triplets, this range changes to $x=[0 ; 0.5]$. In general, the energy drop on the $x>0$ is more pronounced than on the $x$ $<0$ side. Multi-triplet clusters are, therefore, more energetically favorable on the $x>0$ branch of the asymmetry curve. The formation of these large clusters is largely responsible for the occurrence of thermodynamically unstable (twophase) conditions, thereby leading to higher critical temperatures for $x>0$. It is of interest to compare Figs. 2 and 4: The general features of the ground-state-energy follow closely the trends observed in the critical temperature (the open squares in Fig. 2). The energy change involved in the formation of two or three triplet clusters is negative over the entire range of size asymmetry considered here. Multitriplet, neutral ion complexes are favored over isolated triplets, which are effectively attracted to each other.

Of course, the occurrence and structure of multi-ion clusters at low temperatures is not purely energetic and has also an entropic component. The actual average size and structure of the clusters can be further analyzed by inspection 

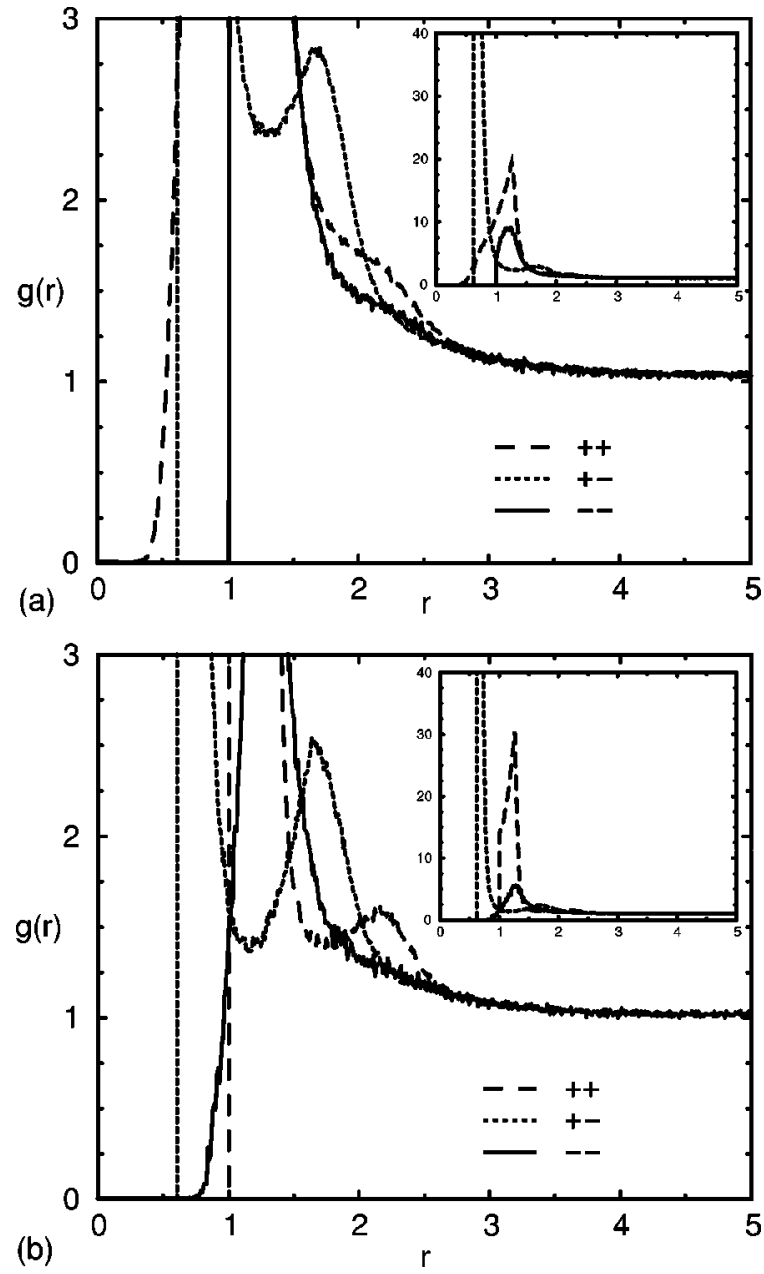

FIG. 5. Radial distribution function $g(r)$ for $2: 1$ spherical electrolytes (a): $x=-0.6, \quad T^{*}=0.028$ and $\rho^{*}=0.02 ; \quad(b): x=0.6, T^{*}=0.044$ and $\rho^{*}$ $=0.02$.

of the,+++- , and -- radial distribution functions. These are shown in Fig. 5 for the 2:1 spherical system with $x$ $=0.6$ and with $x=-0.6$, at approximately the same density $\left(\rho^{*} \approx 0.02\right)$ and at a temperature slightly below the critical $\left(T^{*} \approx 0.97 T_{c}^{*}\right)$. In addition to the expected cation-anion peaks (arising from neutral triplets), both systems exhibit clear like-ion peaks at $r \approx 1.3$, and broad peaks in the range $r=[1.5 ; 3.0]$. All three distribution functions are significantly larger than unity, indicating that even ions carrying a charge of the same sign are effectively attracted to each other. Consistent with our analysis of ground-state-energy clusters, inspection of the distribution functions of the two systems reveals that the system with $x=0.6$ (large divalent cations) exhibits a higher cation-cation first peak and a more pronounced second peak than that of the other system with $x=-0.6$ (small divalent cations). Also note that the actual temperature of the $x=0.6$ system is higher than that of the $x=-0.6$ system (the $x=0.6$ system has a higher critical temperature than the $x=-0.6$ system), thereby reinforcing our view that the larger-cation systems exhibit stronger effective, counterion mediated attractions between like ions.

Interestingly, the phase behavior of dumbbell systems exhibits a number of surprising features that are absent in the spherical systems. The critical temperatures of charged-

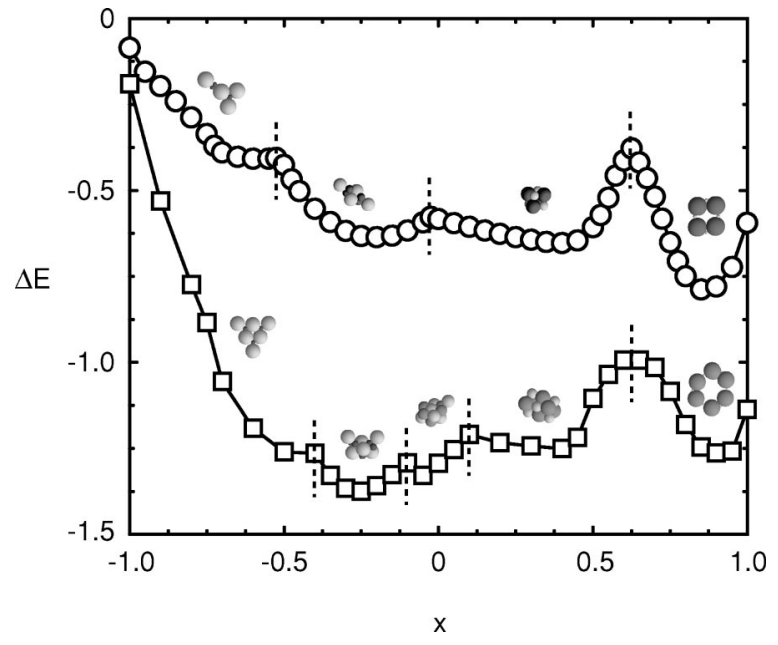

FIG. 6. Ground-state energy of ionic clusters comprising dumbbell cations and spherical anions. Circles and squares correspond to two-triplet and three-triplet clusters, respectively. Several relevant configurations are depicted above the curves; the cations (dumbbells) are shown in dark, and the anions in light. Regions of size asymmetry having distinct ground-state configurations are separated by dashed lines.

dumbbell systems are much lower than those of spherical (2:1) systems. Furthermore, the overall shape of the $T_{c}^{*}$ versus $x$ curve is much more irregular than in the spherical system. Two local maxima can be seen in Fig. 2. The origin of these maxima can also be traced back to the ground-state energy of two-triplet clusters, shown in Fig. 6. Our energy calculations show three relative minima located approximately at $x=-0.25, x=0.4$, and $x=0.9$. The first two of these minima appear to correspond to the two maxima of $T_{c}^{*}$ shown in Fig. 2. The third energy minimum occurs at $x$ $=0.9$; unfortunately, simulations for these highly asymmetric systems are beyond our computational capabilities, and at this point we are unable to establish whether a third $T_{c}^{*}$ maximum occurs or not. On the basis of our ground-state energy analysis, however, we anticipate a third $T_{c}^{*}$ maximum in the vicinity of $x \approx 0.9$.

Overall, for $x>-0.5$, the ground-state energy of dumbbell clusters (about -0.65 at $x=-0.25$ ) is higher than that of spherical clusters (about -0.93 at the minimum), which explains the lower values of $T_{c}^{*}$ for dumbbell systems in the range $x>-0.5$. For $x<-0.5$, as in the spherical case, the cluster energies rise rapidly; we speculate that systems with small dumbbells (i.e., $x \ll-0.5$ ) are relatively stable with respect to systems having larger dumbbells (i.e., $x \approx 0.9$ ) which, as alluded to above, would exhibit higher critical temperatures.

The behavior of the critical density of dumbbells is also markedly different from that observed in spherical systems. A maximum in $\rho_{c}^{*}$ occurs for size-symmetric electrolytes $(x=0)$, and it decreases quite rapidly when the system becomes size-asymmetric. This decrease slows down when $x$ reaches 0.3 , where a shoulder can be seen on the positive side of $x$ (Fig. 3). These results and those for the critical temperature raise questions as to how longer, multi-cation "rods" (e.g., 3:1, 4:1, etc.) or polyelectrolytes behave; does the coexistence region disappear, or is it larger than in spherical, colloidal systems? 


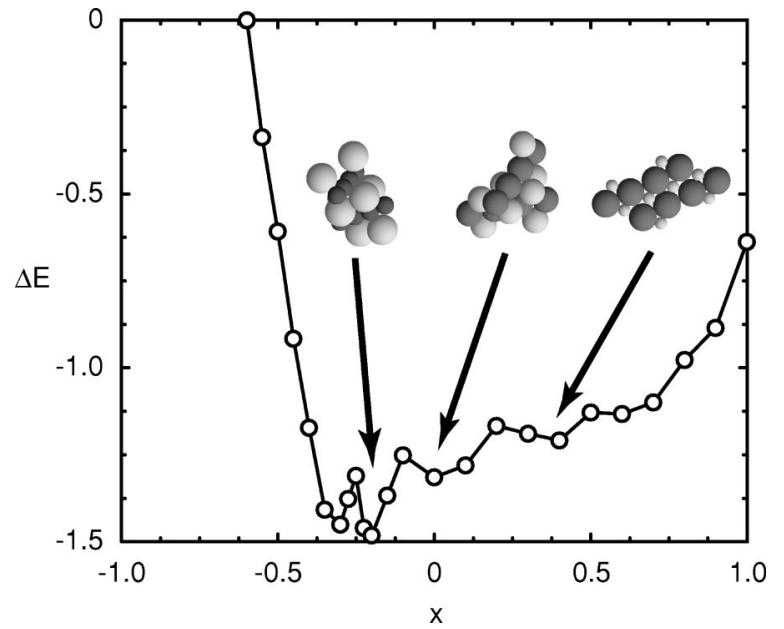

FIG. 7. Ground-state energy of ionic clusters involving rigid, rodlike tetramers (dark) and their respective counterions (light). Several relevant configurations are depicted above the curves.

As a first step to address these questions, we also calculated the ground-state energies of two-complex cluster of charged tetramers and their respective couterions. Figure 7 shows the results as a function of size-asymmetry. The en-

(a)
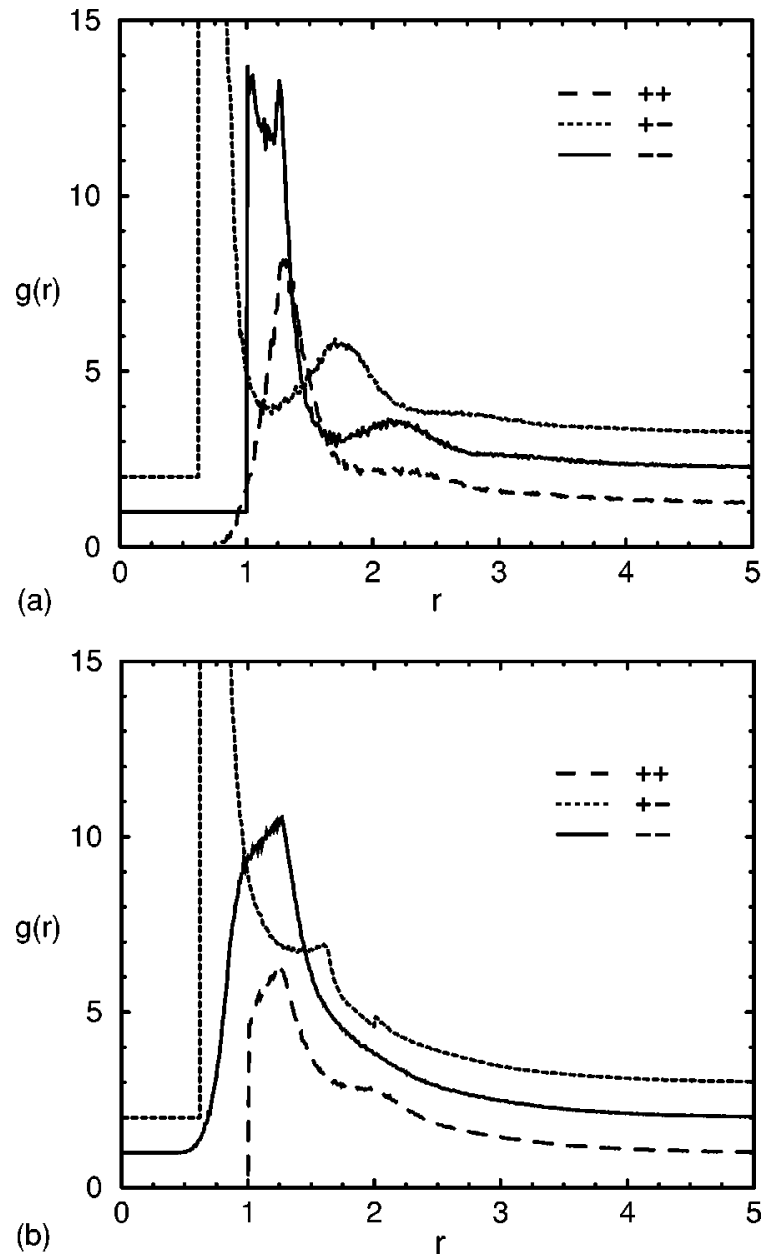

FIG. 8. Radial distribution function $g(r)$ for $2: 1$ dumbbell electrolytes (a): $x=-0.6, T^{*}=0.058$, and $\rho^{*}=0.02 ;(\mathrm{b}): x=0.6, T^{*}=0.057$ and $\rho^{*}$ $=0.02$. For the sake of clarity, the curves for -- and +- distribution have been shifted up by 1 and 2, respectively.

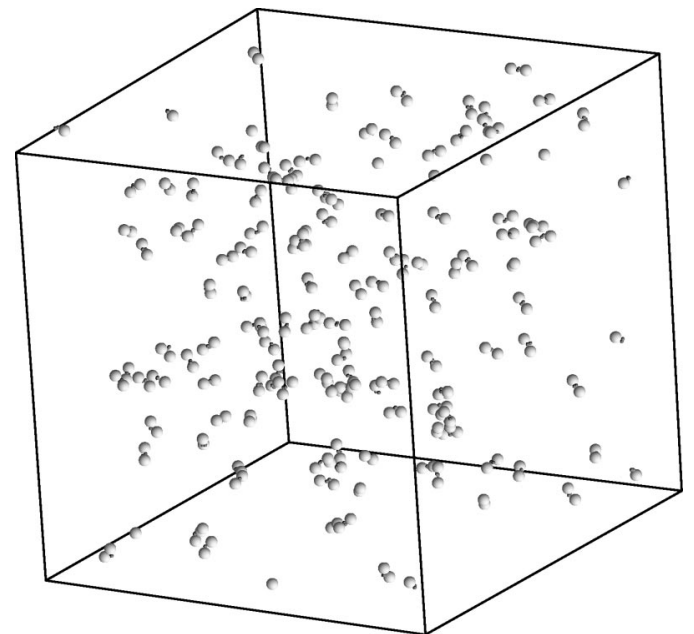

FIG. 9. Typical instantaneous configuration of a 2:1 dumbbell electrolyte $\left(x=-0.6, T^{*}=0.06, \rho^{*}=0.02\right)$.

ergy curve is rugged, with many local minima. This indicates that the low temperature behavior of these systems is likely to be highly sensitive to size asymmetry. A noteworthy feature of the tetramer system is that for $x<-0.6$, bringing together two neutral ion complexes will increase the energy, which suggests that the coexistence region will disappear when $x<-0.6$. The figure also shows how the relative orientation of two tetramer rods changes with size asymmetry. Near the global minimum $(x=-0.2)$, rods in a cluster tend to be perpendicular to each other. However, the angle between two rods decreases as $x$ increases; the rods finally line up side by side when $x$ reaches 0.3 . In contrast, similar calculations using rigid octamers show that rods always tend to line up side by side.

Figure 8 shows radial distribution functions for the 2:1 dumbbell systems with $x=0.6$ and with $x=-0.6$. In contrast to the spherical system, the dumbbell system with $x=0.6$ exhibits a lower peak than the system with $x=-0.6$. This is, however, consistent with the ground-state energy analysis for the dumbbells. As revealed by Fig. 6, the ground-state energy of the system with $x=0.6$ is higher than that of the system with $x=-0.6$. The +- distribution function of the dumbbell system with $x=0.6$ also exhibits another interesting feature: there is a clear well in the curve in the distance range $[1.5,2]$. We attribute this drop to the asymmetric geometry of the dumbbell.

Figure 9 shows a typical instantaneous configuration of a dumbbell electrolyte system with $x=-0.6$ at $T^{*}=0.06$ and $\rho^{*}=0.02$. As expected, almost every cation is associated with two anions, forming a tight, electrically neutral ion triplet. Unlike 1:1 electrolytes, which tend to form large, linear clusters of several dozen ionic pairs, the charge asymmetry of 2:1 systems gives rise to the formation of more complex multi-ion structures having a branched topology. These branched structures are observed in the spherical (Fig. 4) and dumbbell systems.

\section{CONCLUSION}

The critical parameters of spherical, 2:1 electrolytes have been determined as a function of size asymmetry. The 
overall trends observed for the critical temperature and density are in disagreement with available theoretical predictions for the low-temperature phase behavior of electrolytes. It is found that the low temperature phase behavior can be explained in terms of low-energy multi-ion clusters.

Results for shape-asymmetric, dumbbell 2:1 electrolytes reveal additional features which have not been considered theoretically. In particular, in the range $x=[-0.4 ; 0.5]$, the critical temperatures are substantially lower than for spherical systems. Outside this range, the critical temperatures of dumbbells appear to be comparable to those of spherical systems. Two maxima arise in $T_{c}^{*}$, at around $x=-0.25$ and at around $x=0.3$. The coexistence curve is remarkably sensitive to the details of the shape and size of the anions and cations; this fact is also reflected in the ground-state energy of ionic clusters which, depending on size asymmetry, exhibits multiple minima. Simulations of coexistence for large and small ions become increasingly difficult as the asymmetry becomes pronounced. Ground-state energy curves as a function of size-asymmetry appear to provide a reasonable means for anticipating trends in phase behavior.

The results presented in this work could be of importance in the study of colloidal suspensions, particularly in cases where colloidal particles can be represented by collections of charged spheres. Our results could also be important in the study of polyelectrolytes, particularly in situations (e.g., in dilute solutions) where the molecules can be described by a rigid rod of tangent charged spheres. The dumb- bells considered here can only be regarded as colloids or small polyelectrolytes in some highly limiting situations, but they do provide interesting preliminary insights into how phase transitions are likely to evolve as we move towards more general, realistic cases.

\section{ACKNOWLEDGMENTS}

The authors are grateful to A. Z. Panagiotopoulos for a preprint of Ref. 12. Financial support from the National Science Foundation (CTS-9901430) is gratefully acknowledged.

${ }^{1}$ J. W. J. M. Caillol and D. Levesque, J. Chem. Phys. 107, 1565 (1997).

${ }^{2}$ F. O. Orkoulas and A. Z. Panagiotopoulos, J. Chem. Phys. 110, 1581 (1999).

${ }^{3}$ Q. L. Yan and J. J. de Pablo, J. Chem. Phys. 111, 9509 (1999).

${ }^{4}$ J. M. Romero-Enrique, G. Orkoulas, A. Z. Panagiotopoulos, and M. E. Fisher, Phys. Rev. Lett. 85, 4558 (2000).

${ }^{5}$ Q. L. Yan and J. J. de Pablo, Phys. Rev. Lett. 86, 2054 (2001).

${ }^{6}$ Q. L. Yan and J. J. de Pablo, J. Chem. Phys. 114, 1727 (2001).

${ }^{7}$ J. P. Camp and G. N. Patey, J. Chem. Phys. 111, 9000 (1999).

${ }^{8}$ A. M. Ferrenberg and R. H. Swendsen, Phys. Rev. Lett. 61, 2635 (1988).

${ }^{9}$ A. M. Ferrenberg and R. H. Swendsen, Phys. Rev. Lett. 63, 1195 (1989).

${ }^{10}$ A. D. Bruce and N. B. Wilding, Phys. Rev. Lett. 68, 193 (1992).

${ }^{11}$ N. B. Wilding and A. D. Bruce, J. Phys.: Condens. Matter 4, 3087 (1992).

${ }^{12}$ A. Z. Panagiotopoulos and M. E. Fisher, Phys. Rev. Lett. (to be published).

${ }^{13}$ E. González-Tovar, Mol. Phys. 97, 1203 (1999).

${ }^{14}$ A. K. Sabir, L. B. Bhuiyan, and C. W. Outhwaite, Mol. Phys. 93, 405 (1998).

${ }^{15}$ R. R. Netz and H. Orland, Europhys. Lett. 45, 726 (1999). 